\title{
Imaging-documented repeated intratumoral hemorrhage in vestibular schwannoma: a case report
}

\author{
E. S. Mandl • W. P. Vandertop • O. W. M. Meijer • \\ S. M. Peerdeman
}

Received: 1 June 2008 / Accepted: 7 February 2009 /Published online: 3 March 2009

(C) The Author(s) 2009. This article is published with open access at Springerlink.com

\begin{abstract}
Intratumoral hemorrhage in vestibular schwannomas is rare. Symptoms often have an acute onset and include headache, nausea, vomiting, vertigo, and depressed consciousness. Intratumoral hemorrhage is probably caused by vascular fragility associated with tumor characteristics and growth. With hemorrhage in VS being rare, repeated hemorrhage has only been reported twice, and on clinical grounds only. The present report details the case of acute neurological deterioration in a patient with repeated intratumoral hemorrhage inside a vestibular schwannoma with computed tomography and magnetic resonance imaging confirmation. To our knowledge, repeated hemorrhage in vestibular schwannoma with radiological confirmation has not been reported before.
\end{abstract}

Keywords Acoustic neuroma · Schwannoma · Vestibular . Hemorrhage $\cdot$ Radiotherapy

\section{Introduction}

Intratumoral hemorrhage in a vestibular schwannoma is rare [2]. Symptoms often have an acute onset. Intratumoral hemorrhage is probably caused by vascular fragility associ-

E. S. Mandl $(\square) \cdot$ W. P. Vandertop $\cdot$ S. M. Peerdeman

Department of Neurosurgery, 1F-002,

Neurosurgical Center Amsterdam, VU University Medical Center,

7057, Amsterdam 1007 MB, The Netherlands

e-mail: es.mandl@vumc.nl

O. W. M. Meijer

Department of Radiation Oncology,

VU University Medical Center,

Amsterdam, The Netherlands ated with tumor characteristics and growth [1]. Whether extrinsic factors like medication use, and radiotherapy play a role remains unclear [3]. With hemorrhage in VS being rare, repeated hemorrhage has only been reported twice $[4,5]$. We describe acute neurological deterioration in a patient with repeated imaging-documented intratumoral hemorrhage inside a vestibular schwannoma.

\section{Case illustration}

A 59-year old female with left sided sensory hearing loss was referred to our institution in 2001 for treatment of a left-sided vestibular schwannoma (VS). Progressive hearing loss and growth of the VS was the indication for treatment. Our patient opted for fractionated stereotactic radiation therapy (FSRT). Five daily fractions of 5 Gy on the $80 \%$ isodose, encompassing the tumor, were given. At the time of treatment tumor volume measured $9.6 \mathrm{~cm}^{3}$ (Fig. 1a). The past medical history included rheumatoid arthritis treated with methotrexate. During methotrexate treatment the thrombocyte count remained normal. MR imaging in October 2005 showed a round lesion with surrounding hemosiderin deposits within the VS, suggesting previous hemorrhage without clinical evidence (Fig. 1b). With acute onset of headache, ataxia and papiledema in November 2006, a CTscan was performed showing tumor enlargement due to intratumoral hemorrhage, causing an obstructive hydrocephalus (Fig. 1c). The obstructive hydrocephalus was resolved by ventriculoperitoneal (VP) shunt placement. Multiple CT-scans in the following 6 week time period, because VP-shunt malfunction was clinically suspected, showed repeated (sub)acute hemorrhages. The rapid growth of the circumference of the tumor, from 14.3 to $18.6 \mathrm{~cm}^{3}$, due to hematoma enlargement in three months time, necessitated 
Fig. 1 a Axial T1-weighted MR image with gadolinium contrast enhancement showing the left-sided vestibular schwannoma prior to treatment. Maximum tumor volume $9.6 \mathrm{~cm}^{3}$. b Axial T2-weighted MR image of the posterior fossa showing the distinct round lesion inside the VS (arrow) with surrounding hemosiderin deposits. c Axial CT image through the posterior fossa showing a hyperdens area due to intratumoral hemorrhage in the VS. Hematoma diameter was 29 by $23 \mathrm{~mm}$. d Axial T1-weighted postcontrast MR showing the hemorrhage (hypo-intense area) inside the lesion (arrow). The VS enhances homogeneous and contrast-sparing occurs at the location of the hematoma ( $R$ right, $L$ left)
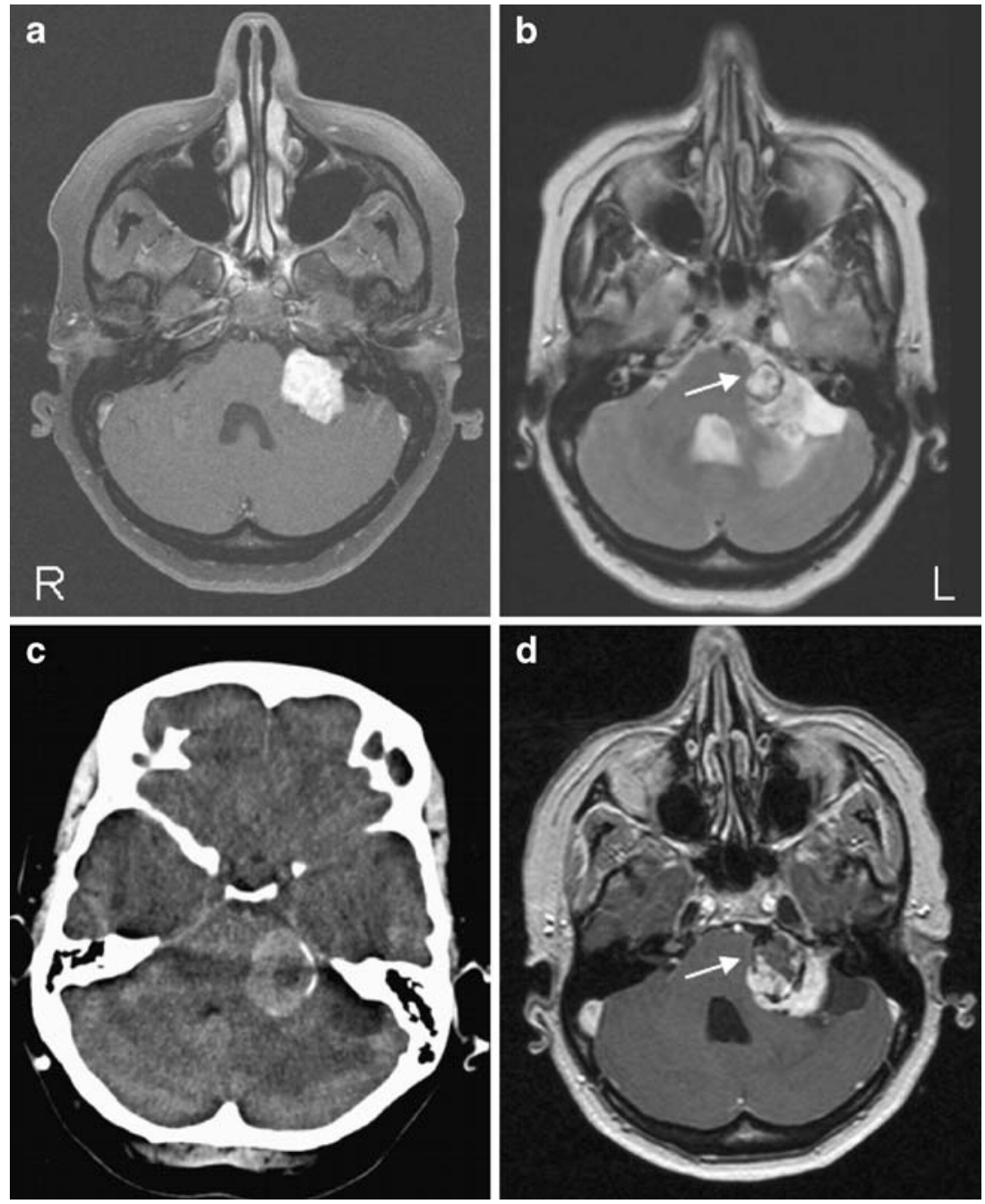

microsurgical excision of the VS (Fig. 1d). A gross total resection was achieved via a retrosigmoid approach. Histopathological examination showed a schwannoma with high vascularity. Post-operatively, the patient had a facial paralysis which on 18 month follow-up improved to a House and Brackmann score V.

\section{Discussion}

Symptomatic intratumoral hemorrhage in a vestibular schwannoma is a rare phenomenon. Our patient suffered multiple hemorrhages in a 16 month time period. Two case reports have been published on repeated intratumoral hemorrhage in VS [4, 5]. The repetitive character of the bleeding in these reports was based solely on acute onset and exacerbation of neurological symptoms without radiological confirmation of this event. These presumed bleedings also occurred in rapid sequence, usually within days, and not during months as in our case. Radiologically confirmed repeated intratumoral hemorrhage has not been described before.

Extrinsic factors that might alter tumor biology and predispose to hemorrhage were the administered radiotherapy and methotrexate. Long standing treatment with methotrexate may lead to trombocytopenia and predispose and maintain intratumoral hemorrhage. Our patient suffered no such adverse effects. Hemorrhage in VS post-radiosurgery has been described. Increased intratumoral pressure through intratumoral infarction or venous obliteration was the presumed pathogenesis for hemorrhage [3]. Comparable pathogenic mechanisms for hemorrhage after the administration of FSRT are likely.

We presented a case with repetitive intratumoral hemorrhages documented on imaging. If the hemorrhaging was due to tumor characteristics alone or in combination with extrinsic factors remains unclear. 
Open Access This article is distributed under the terms of the Creative Commons Attribution Noncommercial License which permits any noncommercial use, distribution, and reproduction in any medium, provided the original author(s) and source are credited.

\section{References}

1. Brady AP, Stack JP (1994) Case report: magnetic resonance demonstration of haemorrhagic acoustic neuroma. Clin Radiol 49:61-63 doi:10.1016/S0009-9260(05)82918-6
2. Chou D, Sampath P, Brem H (1998) Hemorrhagic vestibular schwannoma: an unusual clinical entity. Case report. Neurosurg Focus 5:e9 doi:10.3171/foc.1998.5.3.15

3. Iwai Y, Yamanaka K, Shiotani M, Uyama T (2003) Radiosurgery for acoustic neuromas: results of low-dose treatment. Neurosurgery 53:282-287 doi:10.1227/01.NEU.0000073416.22608.B3

4. Kim SH, Youm JY, Song SH, Kim Y, Song KS (1998) Vestibular schwannoma with repeated intratumoral hemorrhage. Clin Neurol Neurosurg 100:68-74 doi:10.1016/S0303-8467(98)00002-X

5. Kurata A, Saito T, Akatsuka M, Kann S, Takagi H (1989) Acoustic neurinoma presenting with repeated intratumoral hemorrhage. Case report. Neurol Med Chir (Tokyo) 29:328-332 doi:10.2176/nmc.29.328 